\title{
Using 360 VR Video to Improve the Learning Experience in Veterinary Medicine University Degree
}

\author{
Esther Guervós ${ }^{1}$, Jaime J. Ruiz ${ }^{2}$, Pablo Pérez ${ }^{2}$, Juan Alberto Muñoz ${ }^{1}$, César D'az ${ }^{3}$ and Narciso Garc 'a $^{3}$ \\ ${ }^{1}$ Universidad Alfonso X EI Sabio \\ ${ }^{2}$ Nokia Bell Labs \\ ${ }^{3}$ Universidad Politécnica de Madrid
}

\begin{abstract}
We present the results of the Quality of Experience (QoE) evaluation of 360 degree immersive video in university education. Fourth-year Veterinary Medicine students virtually attended some practical lessons which had been recorded in immersive 360 video format, covering topics of Surgical Pathology and Surgery related to horses.

One hundred students participated in the experience. They evaluated it through an extensive questionnaire covering several QoE factors, including presence, audiovisual quality, satisfaction or cybersickness: $79 \%$ evaluated the experience as excellent or good, and they acknowledged an improvement of the learning process by the implementation of VR as didactic tool, and $91 \%$ reported that they would recommend it to other students.

Female students consistently gave slightly better average scores than their male counterparts, although mostly within condence intervals. Strongest inter-gender differences appeared in active social presence dimensions, according to the Temple Presence Inventory. The study also evaluates the suitability of synthetic measurement protocols, such as the Distributed Reality Experience Questionnaire (DREQ) and Net Promoter Score (NPS). We show that NPS is a valid tool for QoE analysis, but that its clustering boundary values must be adapted to the speci cities of the experiment population.
\end{abstract}

\section{Introduction}

We present the results of the Quality of Experience (QoE) evaluation of 360 degree immersive video in university education, in the framework of a pilot project aimed at evaluating the application of Virtual Reality (VR) technology to university lectures. The project involved creating immersive experiences based on $360 \mathrm{VR}$ video for fourth-year university students of Veterinary Medicine in Universidad Alfonso X El Sabio (UAX), Madrid, Spain. The study program includes mandatory practical lessons on horse surgical pathology and surgery at UAX Veterinary Hospital. The exams of those practical lessons occur at the end of the term, which may be weeks or months after the lessons themselves. Through the immersive experiences, students were able to revisit the practical lessons where the surgeon explains all the aspects to consider on the horse surgery process.

The usage of Virtual Reality for education purposes has become more popular in the recent years, due to the drastic cost reduction of VR headsets. Virtual reality is used mostly for adult training in special situations ("vocational training") and in university, significantly in medical fields [1]. For the specific case of veterinary medicine, VR has been reported to be used for anatomy learning [2] or to train laparoscopic skills [3]. There is an increasing number of experiences of the use of VR in education, and this tendency is foreseen to grow in the following years [4].

However, how this technology can fit into a traditional classroom environment is still an open problem, that needs to be carefully addressed for the experience to be effective [5]. On the one hand, the higher level of immersion in the content can enhance the student engagement, thus increasing the teaching-learning performance. On the other, there are some limitations in the technology that can put its benefits under risk: the need for a learning curve, the low visual quality of the displays (that may cause discomfort and cybersickness) or even the fact that the immersion can distract the student from learning $[6,7]$. Therefore it is relevant to evaluate the diverse aspects that influence QoE in VR to understand the potential of this educational approach.

A first element to be considered when evaluating immersive experiences is presence, the sensation of the user to be actively present in the virtual environment or, more generically, the illusion of nonnmediation (the medium of communication, in this case the VR headset and system, becomes transparent to the user) [8]. Presence evaluation has been widely analyzed for the last 20 years, mostly through specific questionnaires. One of the first and most popular ones is the Presence Questionnaire (PQ) [9]. It contains 32 different questions, covering 4 presence factors: Control, Sensory, Distraction and Realism. Along the years, similar questionnaires have been developed, all sharing a similar pattern: tens lots of questions, quite focused on the specific task for which the questionnaire was developed. A good summary can be found in [10]. More recently, there have been efforts to refine questionnaires, either to simplify them or to make them more applicable to a variety of use cases $[11,12]$. In this context, the Temple Presence Inventory [12] provides a comprehensive evaluation of presence through 8 sub-scales, which makes it easily adaptable to different experiments.

In the particular case of omnidirectional 360 degree video, a particularly relevant QoE factor is the audiovisual quality. Subjective assessment of 360 video has been also studied recently, normally by applying traditional audiovisual quality methodologies, such as the Absolute Category Rating (ACR) defined by ITUT P.910 [13], and adapting them to the visualization of videos through Head Mounted Displays (HMDs) [14, 15]. Some variation of this methodology has also been proposed, to adapt to the specificities of HMD omnidirectional video visualization, such as viewing each sequence twice to achieve more stable scores with less visualization time [16]. In any case, the standardization of a protocol for subjective assessment of ominidirectional video is 
still work in progress for scientific and industrial institutions such as the Video Quality Experts Group or the International Telecommunication Union (UIT-T) [17].

A critical element in virtual reality environments is cybersickness: the sickness or discomfort associated to virtual reality experience, which can result in a range of symptoms including nausea, disorientation, headaches, sweating, and eye strain [18]. There are several tools to measure them, both using questionnaries and physiological monotoring of subjects [19], but the most used one is the Simulator Sickness Questionnaire (SSQ) [20].

When considering these tools altogether to evaluate immersive experiences, there is some mismatch among their respective areas of applicability. Presence questionnaires are normally comprised of tens of questions and intended to evaluate full experiences, while quality questions are simple and aimed at repeatedly evaluating short video sequences under different processing types. To fully evaluate a video-based immersive experience, it is not enough to use a simple ACR question, while it might be unfeasible to use a full presence questionnaire. As a result, more compact questionnaries have been proposed to evaluate, for instance, remote operation of machinery using VR [21], or distributed reality experiences [22].

Finally, in this attempt of measure user experience with the least possible number of question, it is worth mentioning the Net Promoter Score (NPS). Coming from marketing analysis, the NPS is based on asking a single question to the subject ('In a scale of 0 to 10 , how probable is that you would recommend it to a colleague or friend?") and, based on it, classifying subjects as promoters $(P$, those who voted 9 or 10$)$, neutral $(N$, those who voted 7 or 8$)$ and detractors ( $D$, those who voted 6 or less). NPS is then computed as [23]:

$$
N P S=100 \% \frac{P-D}{P+D+N}
$$

Even though its reported validity as single predictor of customer loyalty and firm growth is arguable [24], the NPS is still widely used due to its simplicity, and has been adopted to assess satisfaction in health care [25] or education [26], though it normally needs to be complemented with other questions or metrics [27].

\section{Objective}

The main objective of the project was improving the teaching-learning process through the implementation of Virtual Reality technologies. In particular, the project aimed at providing immersive audiovisual experiences to Veterinary Medicine undergraduate students, which could help in the retention of practical lessons when they had no physical access to the veterinary surgery room. It was also relevant for the study that the virtual lessons were part of the regular course, for which the students should be evaluated.

Additionally, we have analyzed the Quality of Experience reported by the students participating in the project, with some specific objectives: analyzing the impact of different presence factors in QoE of 360 video for educational purposes, understading the most relevant elements affecting student satisfaction with the experience, and validating the use of compact questionnaires (with a few questions) in such kind of experiences.

\section{Method \\ Video preparation and delivery}

Some veterinary medicine lessons were recorded for their visualization by students. Content was recorded in the surgery room of the Veterinary Clinic Hospital of UAX, covering some practical lessons of horse surgery (Fig. 1). Some sequences showed preparation for a horse surgery intervention, with the whole surgery team present in the surgery room. Other sequences contained also the students themselves in the practical lessons, including a questions-and-answers session.

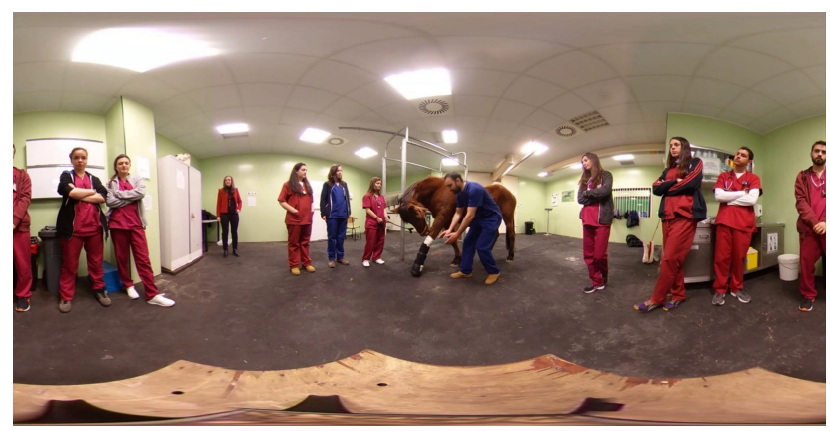

Figure 1. Still picture of the recording from one of the practical lessons at the veterinary surgery room.

Videos were recorded using a Rico Theta V spherical camera, which uses two opposed fisheye lenses with common optical center to obtain a 360 degree view of the scene. The $4 \mathrm{~K}$ equirectangular panorama was generated by the camera software. Afterwards, videos were encoded in HEVC and uploaded into a private server available through the internet. An Android application was developed to list those videos and show them to the students. Once the video was selected in the application, it launched a VR Android player that played it using HTTP Live Streaming. Additionally, the students could also watch the videos as many time as they wanted through a private YouTube channel and at the UAX virtual campus portal (Fig. 2).

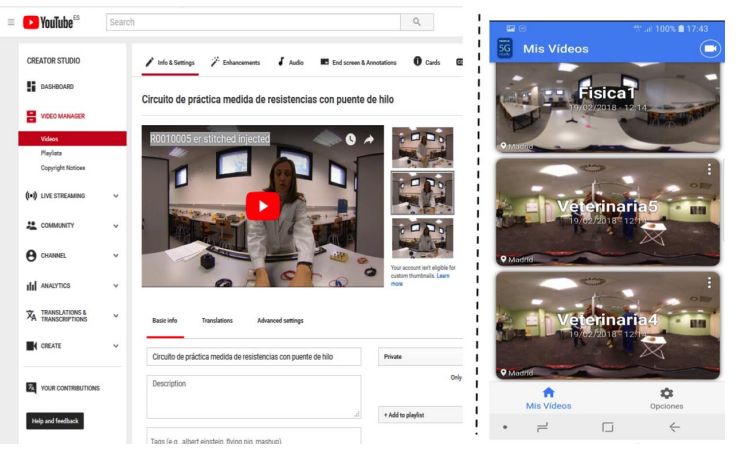

Figure 2. Left: YouTube channel of the lecturer. Right: application for the visualization of the video.

\section{Evaluation}

A pilot group of about 100 fourth-year undergraduate students from the Veterinary Medicine BS degree took part in a formal evaluation of the experience. Each one of them watched the videos using a Samsung Gear VR HMD with an attached Sam- 
sung Galaxy S8+ smartphone, as well as Sennheiser PXC550 wireless headphones, with noise cancellation.

After the experience, they had to fill a questionnaire, composed by the Distributed Reality Experience Questionnaire (DREQ) [22], the Temple Presence Inventory (TPI) [12], and a simplified Simulator Sickness Questionnaire (sSSQ).

DREQ is a short questionnaire designed to evaluate Distributed Reality applications: video-based mixed-reality scenarios where the user is exposed to a combination of her local environment (self-perception and some surrounding objects) and elements from one ore several remote places (in its simplest form, an omnidirectional video). The questionnaire (Table 1) is intended to cover several presence and interaction factors, audiovisual quality, cybersickness and global QoE in a few questions. For this projects, we removed the questions in DREQ about interaction and self-perception, which do not apply. DREQ includes ACR audivisual quality and NPS on its own.

Distributed Reality Experience Questionnaire [22]. QoE factores belong to one of these categories: ${ }^{1}$ Presence (5-point Likert scale), ${ }^{2}$ Media quality (ACR scale [13]), ${ }^{3}$ Cybersicknes (Vertigo scale [28]), and ${ }^{4}$ Quality of Experience. GQOE uses ACR scale and WDRC uses a standard 0-10 probability scale, used to compute Net Promoter Score [23]. .

\begin{tabular}{|l|l|}
\hline Code & Question \\
\hline SPRE $^{1}$ & $\begin{array}{l}\text { I felt like I was actually in the surgery room } \\
\text { I was able to observe the operation as if it hap- } \\
\text { pened around me }\end{array}$ \\
\hline REMQ $^{2}$ & Please rate the perceived quality of the video \\
\hline IECS $^{3}$ & $\begin{array}{l}\text { Did you feel any sickness or discomfort during the } \\
\text { experience? Please rate it } \\
\text { AECS }\end{array}$ \\
\hline GQOE $^{4}$ & $\begin{array}{l}\text { Are you feeling any sickness or discomfort now (af- } \\
\text { ter the experience)? Please rate it } \\
\text { globally? }\end{array}$ \\
WDRC $^{4}$ & $\begin{array}{l}\text { How likely is that you would recommend this expe- } \\
\text { rience to a friend or colleague? }\end{array}$ \\
\hline
\end{tabular}

TPI is a questionnaire developed by Lombard, Ditton and Weinstein [12], based on the knowledge from the state of the art and an extensive validation with more than 500 subjects. It includes the dimensions that address parasocial interactions and social richness as well as the dimensions measured by all of the other existing scales (e.g., spatial presence/transportation, psychological and physical immersion, perceptual realism/naturalness and plausibility or social realism, and engagement/attention). It covers 8 presence factors, each one represented by a variable number of questions ( 3 to 7 ), to a total of 42 items. Those factors are: a) Spatial presence, e.g. presence as "being there"; b) Social presence-actor, the sensation of interaction with the people in the virtual environment; c) Passive social presence, the ability to observe the expressions, voice, etc. from the people in the environment; d) Active social presence, the response of the user (by smiling, loud speaking, etc) to the people in the remote environment; e) Engagement, e.g. mental immersion in the experience, f) Social richness, in which participants are asked to rate their media experience in terms of bipolar word pairs (e.g. "remote" vs "immediate"); g) Social realism, or whether the actions in the virtual scene would occur in the real world; and $h$ ) Perceptual realism, the realism of the sensations (touch, temperature, feeling...). TPI authors propose 7-point scales (normally Likert ones) to cover a wide range of possible responses. To narrow the margin that we provided to the students, we have replaced them by 3-point scales, removing intermediate values.

Finally, to avoid adding 16 extra questions at the end of the test, we have used a simplified version of SSQ (sSSQ) with only three questions, one for each of the groups of symptoms described by SSQ: Oculomotor (headache, eyestrain, difficulty focusing), Disorientation (vertigo, dizziness), and Nausea (stomach awareness, nausea).

To follow a logical structure in the questionnarie, the different sub-questionnaires have been interleaved in the following way: 1) DREQ - presence, 2) TPI, 3) DREQ - media quality and cybersickness, 4) sSSQ, and 5) DREQ - QoE. This way, each of the questions of the short tool (DREQ) is presented just before its longer version (TPI, mSSQ), and the NPS question is left for the last one, so that the students have thought about their experience before answering whether they would recommend it.

\section{Results \\ Presence and quality}

100 students answered to the questions, $75 \%$ female and $25 \%$ male. The unequal gender distribution corresponds to the distribution existing in veterinary studies at UAX.

Figure 3 shows the result of DREQ questions segmented by gender (mean and .95 confidence interval). Average values are between 4 (Good) and 5 (Excellent) to the different questions. There is a tendency of better scores obtained by females with respect to males, even thought it is within confidence intervals, and therefore it might be not significant. To the GQOE question ("how would you rate the quality of your experience globally?"), $35 \%$ rated the experience as excellent, $43 \%$ as good and $22 \%$ as normal. meaning that there is a global feeling of satisfaction with it.

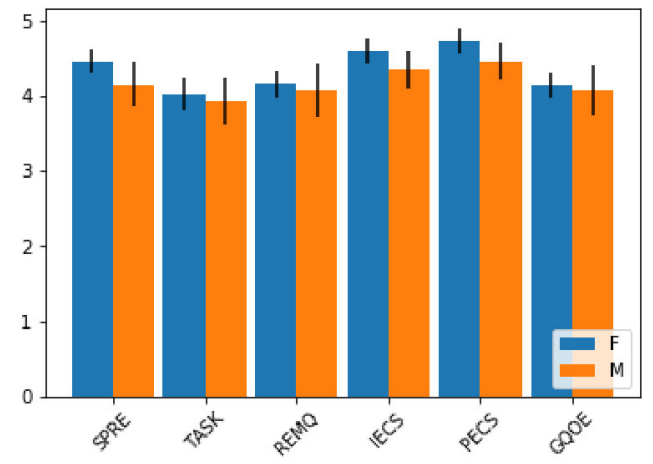

Figure 3. DREQ results by gender.

Fig. 4 shows the distribution of the presence questions, segmented by gender. To be able to present them synthetically, we have created a composite index for each presence factor, by averaging all the questions in each category and normalizing the results between 1 (positive presence) and -1 (negative presence). The most significant difference comes from the "Social Active" 
presence, where females report active responses to the people in the video (smiling, speaking to them, etc) while males do not.

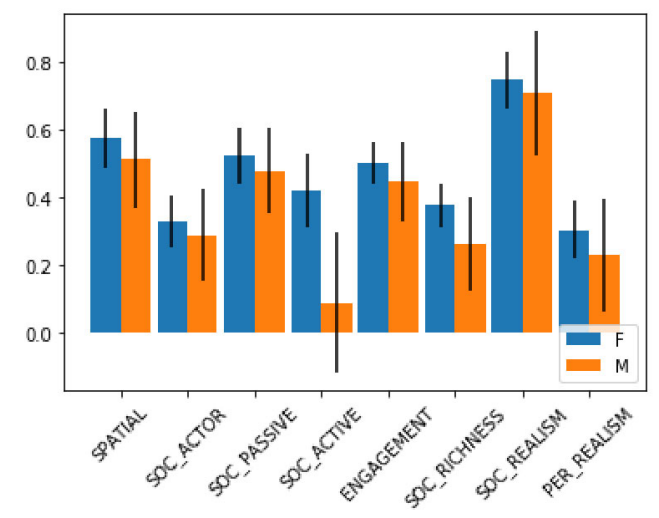

Figure 4. TPI results by gender.

\section{Cybersickness}

Cybersicknes scores are particularly good (Fig. 5). Very few users reported any sickness at all, and average levels of inexperience and post-experience sickness are low. This can be due to the fact that the videos were recorded from a still position without any camera motion, which is known to be the main source of sickness in immersive video [28].

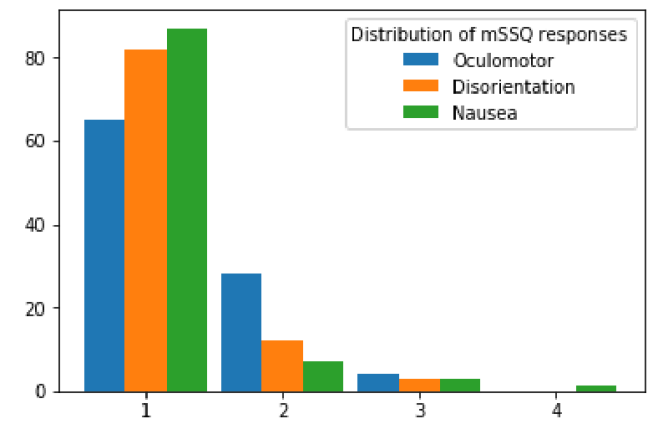

Figure 5. Results of $m S S Q$.

Table 2 shows Pearson cross-correlation coefficients between the different mSSQ factors (OCULomotor, DISOrientation, and NAUSea) and the in-experience and post-experience cybersickness questions from DREQ (IECS and PECS respectively). It is worth noting that $\mathrm{mSSQ}$ responses measure sickness level (low is good), while DREQ measures comfort level (low is bad), and therefore cross-correlations have negative signs.

Correlation between $\mathrm{MSSQ}$ and DREQ cybersickness factors.

\begin{tabular}{lrrrrr}
\hline & IECS & PECS & OCUL & DISO & NAUS \\
\hline IECS & 1.00 & 0.72 & -0.52 & -0.49 & -0.13 \\
PECS &. & 1.00 & -0.57 & -0.60 & -0.30 \\
OCUL &. &. & 1.00 & 0.53 & 0.34 \\
DISO &. &. &. & 1.00 & 0.36 \\
NAUS &. &. &. &. & 1.00 \\
\hline
\end{tabular}

\section{Net Promoter Score}

Distribution of answers to WDRC question ("Would you recommend this experience to a friend or colleague?") is shown in Fig. 6. According to the original definition, this would provide a NPS value of $14 \%$. This is good, although not excellent, and may be a bit low compared to the responses to DREQ questions, where similar quality numbers provided NPS values in the range of 30 to 40 percent [22].

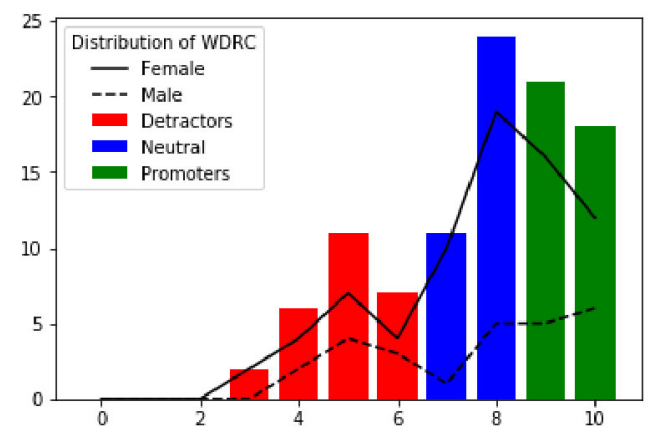

Figure 6. WDRC results by gender.

However, Fig. 7 shows an interesting pattern: WDRC responses of an 8 have normally better quality values than a 9. This might be important in the cultural environment of university qualifications in Spain, were an 8 is perceived as a good feedback to the experiment.

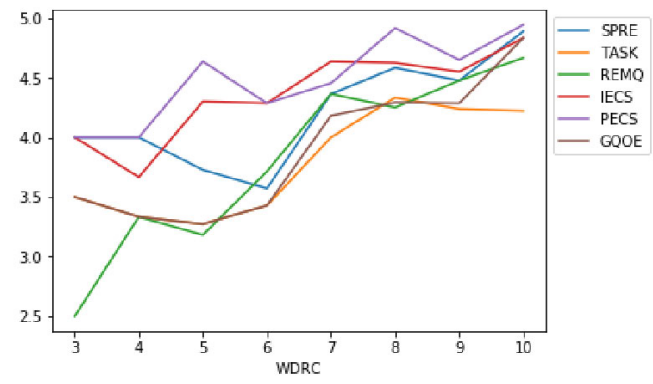

Figure 7. $D R E Q$ values for each WDRC response.

With this in mind, we have clustered the student responses to WDRC differently from the original NPI recommendation: detractors point 5 or less, 6 and 7 are neutral, and 8 to 10 are supporters. This new clustering allows better identification of quality and presence factors in the student satisfaction, as shown in Figs. 8 and 9, which cluster DREQ and TPI responses by NPS category. In terms of DREQ factors, audiovisual quality and global QoE show the strongest differences between detractors and supporters. In terms of presence, social active and social realism seem to be the most relevant factors. Under this clustering, NPS score of this experiment rises up to $44 \%$. In any case, comparing NPS values between experiments should be done with care, as underlying WDRC answers might be biased by the subject assumptions about what a good feedback is.

\section{Conclusions}

In the project we have integrated VR technologies into the existing practical lessons of university Veterinary Medicine stud- 


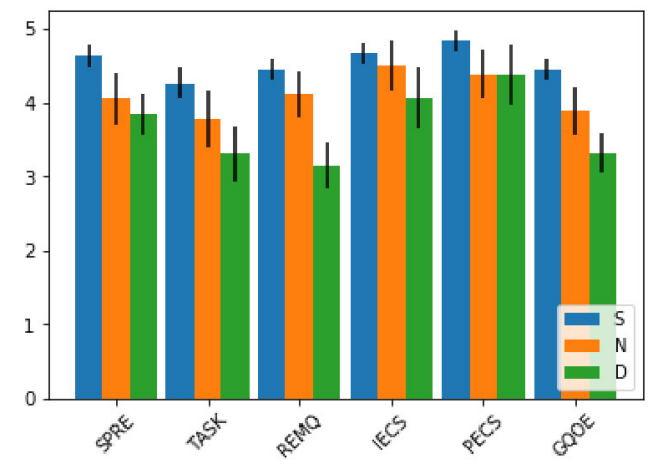

Figure 8. DREQ results by modified NPS categories.

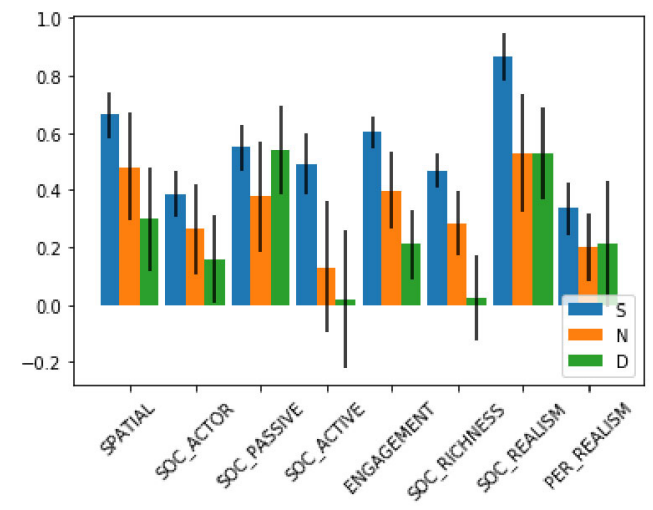

Figure 9. TPI results by modified NPS categories.

ies. This fulfills the main objective of the project, which is verifying that the immersive experience facilitates the teaching-learning experience, by effectively removing the barrier between the student, the lecturer and the machine. Most students felt spatially present in the surgery room, thus perceiving the scene as if they were actually there in the moment of the surgical intervention.

We have found that responses to WDRC question according to the standard NPS score may be misleading, as they could consider 8 responses as coming from neutral subjects, while their responses match better with a supporter behavior. Therefore we have adapted the NPS score classification to better reflect this effect.

We have analyzed quality, presence and sickness factors in the experience, segmenting both by gender and by (modified) net promoter score category (detractor, neutral, supporter). DREQ scores have average values between 4 and 5 , with slightly better scores in female students. Presence factors have a higher variation. Social presence seem to have higher discriminative power with respect to Net Promoter Score than what spatial presence has. No significant cybersickness has been reported.

\section{Acknowledgments}

This work has been partially supported by Spain's Centre for the Development of Industrial Technology (CDTI) under project IDI-20180015 (VINEDO), and by the Ministerio de Ciencia, Innovación y Universidades (AEI/FEDER) of the Spanish Government under project TEC2016-75981 (IVME).

\section{References}

[1] L. Freina and M. Ott, "A literature review on immersive virtual reality in education: State of the art and perspectives.," eLearning \& Software for Education, no. 1, 2015.

[2] J. H. Seo, B. M. Smith, M. Cook, E. Malone, M. Pine, S. Leal, Z. Bai, and J. Suh, "Anatomy builder vr: applying a constructive learning method in the virtual reality canine skeletal system," in International Conference on Applied Human Factors and Ergonomics, pp. 245-252, Springer, 2017.

[3] B. A. Fransson, C.-Y. Chen, J. A. Noyes, and C. A. Ragle, "Instrument motion metrics for laparoscopic skills assessment in virtual reality and augmented reality," Veterinary surgery, vol. 45, no. S1, pp. O5-O13, 2016.

[4] S. A. Becker, M. Cummins, A. Davis, A. Freeman, C. G. Hall, and V. Ananthanarayanan, "Nmc horizon report: 2017 higher education edition," tech. rep., The New Media Consortium, 2017.

[5] ClassVR, "White paper: A guide to AR \& VR in education." Online, Jun 2017. http://www.classvr.com/download/whitepaper-a-guideto-ar-vr-in-education/.

[6] A. B. Ray and S. Deb, "Smartphone based virtual reality systems in classroom teaching - a study on the effects of learning outcome," in Technology for Education (T4E), 2016 IEEE Eighth International Conference on, pp. 68-71, IEEE, 2016.

[7] M. A. Rupp, J. Kozachuk, J. R. Michaelis, K. L. Odette, J. A. Smither, and D. S. McConnell, "The effects of immersiveness and future vr expectations on subjective experiences during an educational 360 video," in Proceedings of the Human Factors and Ergonomics Society Annual Meeting, vol. 60, pp. 2108-2112, SAGE Publications Sage CA: Los Angeles, CA, 2016.

[8] M. Lombard and T. Ditton, "At the heart of it all: The concept of presence," Journal of Computer-Mediated Communication, vol. 3, no. 2, 1997.

[9] B. G. Witmer and M. J. Singer, "Measuring presence in virtual environments: A presence questionnaire," Presence, vol. 7, no. 3, pp. 225-240, 1998.

[10] J. Van Baren, "Measuring presence: A guide to current measurement approaches," Deliverable of the OmniPres project IST-2001-39237, 2004.

[11] T. Hartmann, W. Wirth, H. Schramm, C. Klimmt, P. Vorderer, A. Gysbers, S. Böcking, N. Ravaja, J. Laarni, T. Saari, et al., "The spatial presence experience scale (SPES)," Journal of Media Psychology, 2015.

[12] M. Lombard, T. B. Ditton, and L. Weinstein, "Measuring presence: the temple presence inventory," in Proceedings of the 12th Annual International Workshop on Presence, pp. 1$15,2009$.

[13] ITU-T, "P.910: Subjective video quality assessment methods for multimedia applications," 042008.

[14] E. Upenik, M. Rerabek, and T. Ebrahimi, "Testbed for subjective evaluation of omnidirectional visual content," in 32nd Picture Coding Symposium (PCS), Nuremberg, Germany, December 4-7, 2016., no. EPFL-CONF-221560, IEEE, 2016.

[15] R. Schatz, A. Sackl, C. Timmerer, and B. Gardlo, "Towards 
subjective quality of experience assessment for omnidirectional video streaming," in Proc. 9th Int. Conf. Qual. Multimedia Exp.(QoMEX), pp. 1-6, 2017.

[16] A. Singla, S. Fremerey, W. Robitza, P. Lebreton, and A. Raake, "Comparison of subjective quality evaluation for hevc encoded omnidirectional videos at different bit-rates for uhd and fhd resolution," in Proceedings of the on Thematic Workshops of ACM Multimedia 2017, pp. 511-519, ACM, 2017.

[17] ITU-T, "P.360-VR: Subjective test methodologies for 360 degree video on HMD (Under Study)," 2018.

[18] J. J. LaViola Jr, "A discussion of cybersickness in virtual environments," ACM SIGCHI Bulletin, vol. 32, no. 1, pp. 47$56,2000$.

[19] S. Davis, K. Nesbitt, and E. Nalivaiko, "A systematic review of cybersickness," in Proceedings of the 2014 Conference on Interactive Entertainment, pp. 1-9, ACM, 2014.

[20] R. S. Kennedy, N. E. Lane, K. S. Berbaum, and M. G. Lilienthal, "Simulator sickness questionnaire: An enhanced method for quantifying simulator sickness," The international journal of aviation psychology, vol. 3, no. 3, pp. 203220, 1993.

[21] K. Brunnström, M. Sjöström, M. Imran, M. Pettersson, and M. Johanson, "Quality of experience for a virtual reality simulator," in Human Vision and Electronic Imaging (HVEI), Burlingame, California USA, 28 January-2 February, 2018, 2018.

[22] A. Villegas, P. Perez, E. Gonzalez-Sosa, R. Kachach, and J. Ruiz, "Towards a Distributed Reality: a multi-video approach to xR," in Proc. IEEE VR, Jan 2019. (submitted).

[23] F. F. Reichheld, "The one number you need to grow," Harvard business review, vol. 81, no. 12, pp. 46-55, 2003.

[24] T. L. Keiningham, B. Cooil, T. W. Andreassen, and L. Aksoy, "A longitudinal examination of net promoter and firm revenue growth," Journal of Marketing, vol. 71, no. 3, pp. 39-51, 2007.

[25] D. Hamilton, J. V. Lane, P. Gaston, J. Patton, D. Macdonald, A. Simpson, and C. Howie, "Assessing treatment outcomes using a single question: the net promoter score," The bone \& joint journal, vol. 96, no. 5, pp. 622-628, 2014.

[26] K. Palmer and C. Devers, "An evaluation of mooc success: Net promoter scores," in EdMedia+ Innovate Learning, pp. 1648-1653, Association for the Advancement of Computing in Education (AACE), 2018.

[27] M. W. Krol, D. de Boer, D. M. Delnoij, and J. J. Rademakers, "The net promoter score-an asset to patient experience surveys?," Health Expectations, vol. 18, no. 6, pp. 30993109, 2015.

[28] P. Perez, N. Oyaga, J. J. Ruiz, and A. Villegas, "Towards systematic analysis of cybersickness in high motion omnidirectional video," in Proc. 10th Int. Conf. Qual. Multimedia Exp. (QoMEX), 2018.

\section{Author Biography}

Esther Guervós received her BS in Physics from Universidad de Salamanca (1994), her PhD in Industrial Engineering from Universidad Nacional de Educación a Distancia (2007) and her Master in Renewable Energy and the Energy Market by Escuela de Organización Industrial (2003). Since 2009 she has been a member of the faculty of Universidad Alfonso X el Sabio, where she is now Associate Professor with ACAP certi cation (Accreditation and Prospective Agency of the Universities of Madrid).

Jaime J. Ruiz received his Telecommunication Engineering degree (integrated BS-MS) from Universidad Politécnica de Madrid (1993), and his PhD in Industrial Engineering from Universidad Nacional de Educación a Distancia (2012). He holds also a Master in Marketing and Commercial Management from ESIC, Madrid, Spain. He has leaded several R\&D projects, and has a deep experience and knowledge on video performance solutions involving distributed storage and streaming technology. His research interest include low latency and high-performance video storage and streaming, mainly focused on 360 and immersive VR video.

Pablo Pérez received his Telecommunication Engineering degree (integrated BS-MS) in 2004 and his PhD in Telecommunication Engineering in 2013 (Doctoral Graduation Award), both from Universidad Politécnica de Madrid. He is currently Senior Researcher in the Distributed Reality Solutions department at Nokia Bell Labs, as well as vice-chair of the Immersive Media Group of the Video Quality Experts Group (VQEG). His research interests include multimedia Quality of Experience, video transport networks, and immersive communication systems.

Juan Alberto Muñoz received his BS in Veterinary Medicine from Universidad Complutense de Madrid in 2013, and MS (2014) and PhD (2017) in Veterinary Science by Universidad Alfonso X el Sabio (UAX). He has been lecturer in the Veterinary School of Lyon, France (2008) and he is now member of the faculty of UAX. He is chief of the large animal surgery service in UAX Veterinary Clinic Hospital, as well as member of the Examination Committee of the European College of Veterinary Surgeons.

Cesar Díaz received his Telecommunication Engineering degree (integrated BS-MS) in 2007 and his PhD. degree Telecommunication Engineering in 2017, both from Universidad Politécnica de Madrid (UPM). Since 2008 he has been a member of the Image Processing Group of the UPM, where he has been actively involved in Spanish and European projects. His research interests are in the area of multimedia delivery, video transmission protection and immersive communication systems.

Narciso García received his Ingeniero de Telecomunicacin degree ( ve years engineering program) in 1976 (Spanish National Graduation Award) and his his PhD in Communications in 1983 (Doctoral Graduation Award), both from the Universidad Politécnica de Madrid (UPM). Since 1977, he has been a member of the faculty of the UPM, where he is currently a Professor of Signal Theory and Communications. He leads the Image Processing Group of UPM. His current research interests include digital image and video compression and computer vision. He received the Junior and Senior Research Awards of the Universidad Politécnica de Madrid in 1987 and 1994, respectively. 


\section{JOIN US AT THE NEXT EI!}

IS\&T International Symposium on

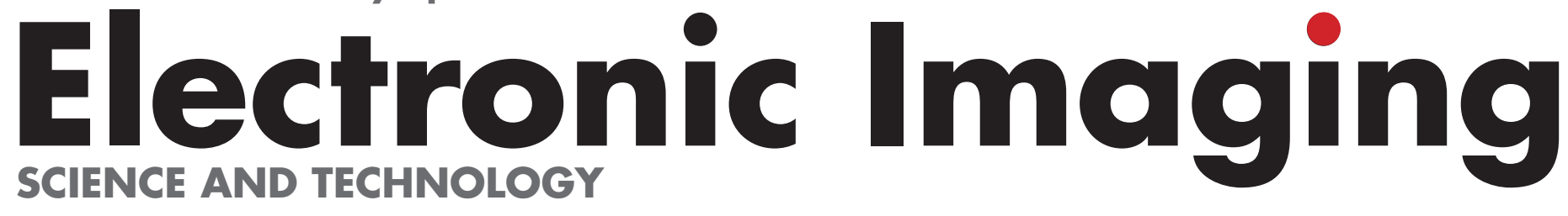

Imaging across applications ... Where industry and academia meet!
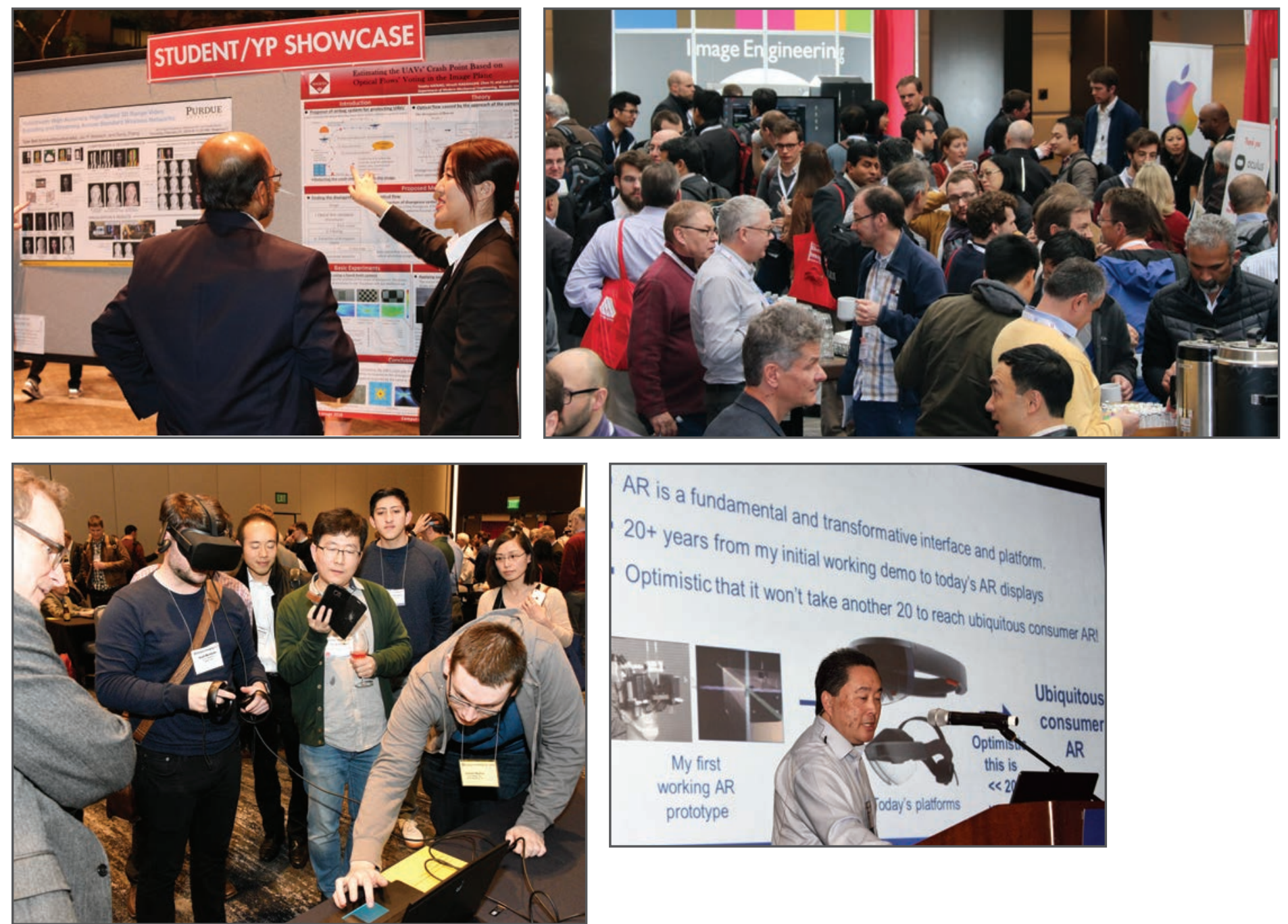

- SHORT COURSES • EXHIBITS • DEMONSTRATION SESSION • PLENARY TALKS •

- INTERACTIVE PAPER SESSION • SPECIAL EVENTS • TECHNICAL SESSIONS •

www.electronicimaging.org

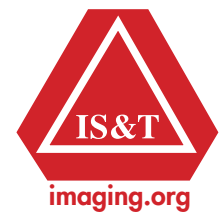

